\title{
Enquête judiciaire, littérature et imaginaire social au XIXe siècle
}

\author{
Dominique KaLIFA \\ Université Paris I \\ Dominique.Kalifa@univ-paris1.fr
}

Recibido: $15 / 03 / 2011$

Aceptado: 07/07/2011

\section{RESUMEN:}

La cultura occidental posee una larga tradición de causas célebres, de hechos y biografías de criminales famosos. En el siglo XIX se produce una doble inflexión en esta tradición: primero, el interés se desplaza hacia la estadística judicial, los datos del juicio y sus principales actores. En segundo lugar, la codificación de este cambio incorpora un nuevo imaginario social. Las etapas de este proceso son seguidas a través de la sociología de la literatura decimonónica.

Palabras clave: literatura criminal, imaginario social, proceso judicial, representación, modernidad.

Juridical Investigacion, Literature and Social Imaginary in the 19th Century

\begin{abstract}
:
Western culture has a long tradition of celebrated causes, facts and biographies of famous criminals. The nineteenth century produced a double inflection in this tradition: first, interest shifted to the judicial statistics, data from the trial, and its main actors. Secondly, the codification of this change includes a new social imaginary. This process is followed through the sociology of nineteenth-century literature.
\end{abstract}

Key words: criminal history, social imaginary, courts records, representation, modernity.

Summary: Introduction. 1. L'avènement du roman de l'enquête. 1.1 Fondements. 1.2 Frémissements. 1.3 Formalisations. 2. L'institution d'un imaginaire. 2.1 Esprit scientifique? 2.2 Sociologie et hiérarchies romanesques. 2.3 Appropriations.

\section{Introduction}

Le XIXe siècle n'a pas inauguré la rencontre entre la justice et la littérature. Une ancienne et abondante tradition de «causes célèbres», de factums et de biographies de criminels s'est développée en Europe depuis la fin du Moyen Age et a nourri une large part de la culture occidentale ${ }^{1}$. Mais le XIXe siècle a donné à cette production une double et décisive inflexion. La première concerne les contenus du récit: du crime, du procès, de l'exécution, scènes traditionnelles de la représentation littéraire, l'intérêt s'est peu à peu déplacé vers l'enquête judiciaire, ses procédures et ses

${ }^{1}$ GEREMEK, Bronislaw: Les Fils de Caïn. L'image des pauvres et des vagabonds dans la littérature européenne du XVe au XVIIe siècle (1980), Paris, Flammarion, 1991. 
acteurs. Un prolixe imaginaire en a résulté, dont les figures ont rapidement débordé le registre littéraire pour affecter l'ensemble de la production fictionnelle: imagerie, théâtre, journal, cinéma, etc. Une seconde mutation en procède : loin de se limiter à une intrigue ou à des thématiques à la mode, cette convergence entre la fiction et l'enquête s'est rapidement codifiée, jusqu'à se constituer en genre (le «policier»), le seul genre d'ailleurs, avec le poème en prose, que la modernité ait inventé, et dont le succès n'a pas désarmé depuis ${ }^{2}$. Tardive et imparfaite, cette codification n'épuise évidemment pas les représentations du crime et de la justice, mais la dynamique de l'enquête constitue un ressort puissant, qui anime tout un imaginaire. On rappellera dans un premier temps les grandes étapes qui scandent l'avènement du récit d'investigation, avant d'en évoquer les principaux usages et les fonctions sociales.

\section{L'avènement du roman de l'enquête}

\subsection{Fondements}

Anciens et prolifiques l'un comme l'autre, deux grands types d'ouvrages alimentent au début du XIXe siècle une ample littérature du crime et du châtiment. Le premier se destine plutôt à un public lettré auquel il offre de longue date, sous forme de «causes célèbres» et de factums, de minutieux récits de procès qui exploitent la curiosité pour le crime, entretiennent la familiarité avec la chose judiciaire, mais permettent parfois aussi d'exposer des questions plus sensibles. On sait ainsi que leur multiplication au siècle des Lumières a accompagné la critique de l'arbitraire et contribué à la genèse de la sphère publique moderne ${ }^{3}$. L'autre type emprunte les réseaux du colportage et nourrit une vaste production d'imprimés à bas prix: récits de faits divers portés par une multitude d' «occasionnels», de canards et de broadsheets, biographies romancées de criminels, relations d'exécution capitale. Présente dans toute l'Europe depuis la fin du Moyen $\mathrm{Age}^{4}$, cette «littérature de la gueuserie» a assez tôt signalé son caractère hybride et polysémique: l'impératif de moralisation, explicite au travers de la confession et du châtiment du malfaiteur, y croise en permanence l'affirmation d'un destin exceptionnel et héroïque. C'est le cas d'une large part de la tradition picaresque espagnole, de bien des récits véhiculés en France par la célèbre «Bibliothèque bleue» de Troyes (histoires de Mandrin, Cartouche, compère Guilleri, etc.), ou du très prolifique Newgate Calendar, qui réunit depuis le XVIIIe siècle les biographies de criminels anglais recueillies par le chapelain de la prison de Newgate. Mais l'enquête judiciaire n'occupe dans ces récits qu'une place très marginale. Ce qu'ils mettent en scène, c'est le destin aventureux des criminels et des brigands fameux, les crimes nombreux qu'ils ont commis, le procès

\footnotetext{
${ }^{2}$ DUBOIS, Dubois: Le Roman policier ou la modernité, Paris, Nathan, 1992.

${ }^{3}$ MAZA, Sarah: Private Lifes and Public Affairs; the "Causes Célèbres" of the Pre-Revolutionary France, Berkeley, University of California Press, 1993.

4 DU SORBIER, Françoise: Récits de gueuserie et biographies criminelles de Head à Defoe, Paris, Didier, 1985; CHARTIER, Roger, Lecteurs et lectures dans la France d'Ancien Régime, Paris, Seuil, 1987 .
} 
qui les condamne et surtout le châtiment final qui semble lier, au pied de l'échafaud, le repentir, l'expiation et la rédemption héroïque.

Or, loin d'être archaïques, ces récits connaissent au XIXe siècle leurs plus belles heures. Constamment réédités et adaptés, ils bénéficient de l'extension des circuits du colportage qui atteignent leur apogée vers le milieu du siècle. Ils parviennent surtout à se diversifier et à se renouveler. En Angleterre, cette literature of roguery se prolonge au travers des feuilletons à succès de William Ainsworth, dont le Rookwood (1834), puis le Jack Sheppard (1839), actualisent les aventures de bandits de grand chemin ${ }^{5}$. D'autres auteurs, comme Edmund Bullwer-Lytton (Paul Clifford, 1830; Eugen Aram, 1832) ou William Gilmore (Martin Faber, the story of a criminal, 1833) s'attachent à investir d'un caractère plus social ces biographies de criminels. En France, c'est surtout par l'intermédiaire du mélodrame que se ressource la tradition du bandit de grand chemin, comme en témoigne l'immense succès de L'Auberge des adrets (1823) et de son héros Robert Macaire, qui accède au type sous la monarchie de Juillet. Mâtinée de roman gothique, c'est aussi au travers des feuilletons des bas-fonds que se perpétue cette tradition, en France (Les Mystères de Paris d'Eugène Sue en 1842-43, Les Mystères de Londres de Paul Féval en 1843) comme en Angleterre où George Williams Reynolds publie dès 1844 l'effrayante série des Mysteries of London, puis of the Court of London. Tout comme les innombrables avatars qui leur font suite, ces grands feuilletons criminels jonglent avec les assassins, les misérables, les victimes, les justiciers, mais ne font guère de cas des procédures judiciaires de l'enquête.

\subsection{Frémissements}

Quelques signes, apparus vers la fin de la décennie 1820, laissaient pourtant présager une évolution en ce sens. Le premier concerne l'invention du policier comme figure littéraire. La fonction ressortissait jusque là des basses besognes du service de l'État et nul n'avait songé à l'investir d'une dimension romanesque; le personnage était trivial, la fonction honteuse et tout en lui inspirait le mépris. Publiés en 1828, les Mémoires de Vidocq inaugurent en ce sens un transfert capital, tant au regard de la littérature qu'ils dotent d'un personnage appelé à devenir un puissant organisateur textuel, qu'à celui de la police, engagée dès lors dans un lent procès de requalification. Bien qu'apocryphes, ces souvenirs gagnent largement leur pari: transposer au policier l'aura romanesque jusque là réservée au criminel. Certains auteurs comprirent d'emblée l'énorme portée de ce basculement. C'est le cas de Balzac qui, outre les nombreux avatars de Vidocq dont il peuple ses romans, introduit dès 1841 «le fameux Corentin, le bras droit de Fouché» et son acolyte Peyrade dans Une ténébreuse affaire. Mais Balzac entrevoit surtout le pouvoir narratif de l'enquête judiciaire. Les investigations qu'il mène personnellement en 1839 pour pallier les lacunes de l'instruction dans l'affaire Peytel ${ }^{6}$ l'ont

${ }^{5}$ HOLLINGSWORTH, Keith: The Newgate Novel, 1830-1847: Butler, Ainsworth, Dickens and Thackeray, Detroit, Wayne State University Press, 1963.

${ }^{6}$ BAUDOUIN, Patricia: «Justice, presse et politique. L'engagement de Balzac dans l'affaire Peytel», Revue d'histoire du XIXe siècle, n 26-27, 2003, pp. 331-348. 
convaincu de l'importance de l'opération et du pouvoir des magistrats, qu'il est aussi un des premiers à mettre en scène (le juge d'instruction Popinot dans L'Interdiction, le juge Camusot dans Splendeurs et misère des courtisanes).

Le mouvement est synchrone en Grande-Bretagne. Dès 1827 sont publiées les Scenes in the Life of a Bow Street Runner (by Richmond), et les Mémoires de Vidocq y sont immédiatement traduits ${ }^{7}$. L'intrication entre souvenirs et littérature y est également comparable. En 1852 paraissent ainsi les célèbres Recollections of a Detective Police-Officer qu'écrit, sous le pseudonyme de Waters, le romancier victorien William Russel ${ }^{8}$. Au même moment, Dickens, qui déjà avait introduit les inspecteurs Blathers et Duff, deux Bow Street Runners sans éclat, dans Oliver Twist en 1837, met en forme en 1850 plusieurs récits et anecdotes de détectives londoniens, ainsi que la relation de ses pérégrinations nocturnes avec l'inspecteur Field de Scotland Yard'. Deux ans plus tard, il livre dans Bleak House (1852-1853) le premier enquêteur de police véritablement consistant de la littérature britannique, le sergent Buckett. Entré par petite touches dans le roman, il en devient rapidement le principal ordonnateur, qui en relie tous les fils et en élucide toutes les intrigues.

Mais cette entrée du policier en littérature se double, là aussi dès la fin des années 1820 , de la diffusion d'un nouvel imaginaire, celui de la «frontière» et de la «prairie», qu'introduit en Europe l'immense succès des romans de l'Américain Fenimore Cooper. Fascinant les contemporains, l'univers des sauvages, de la traque, de la piste, est rapidement transposé dans celui de la ville. «Paris, voyez-vous, est comme une forêt du Nouveau Monde», explique Balzac en $1835^{10}$, tandis qu'Eugène Sue inscrit explicitement ses Mystères de Paris dans le sillage des Indiens de Cooper. Quelques années plus tard, Féval intitule «La forêt de Paris» un des chapitres de ses Habits noirs ${ }^{11}$. La greffe est d'autant plus rapide qu'elle recoupe l'obsession du crime et le désir d'exploration sociale dont la période est porteuse. La figure du «limier de police», qu'enregistre en 1835 le Dictionnaire de l'Académie française ${ }^{12}$, et l'intérêt romanesque pour l'enquête judiciaire, sont le produit de cette convergence, dont la diffusion est rapide dans la littérature feuilletonesque. Dans Les Mohicans de Paris, publiés par Dumas de 1854 à 1859, le héros Salvator, accompagné du chien Brésil, part ainsi «la chasse à la vérité» tandis que le chef de la Sûreté Jackal mène de très subtiles investigations de terrain, déchiffrant les indices et les traces pour construire à leur aune de subtiles hypothèses.

\footnotetext{
${ }^{7}$ Memoirs of Vidocq, Principal Agent of the French Police until 1827, London, Hunt and Clarke, 1828.

${ }^{8}$ Recollections of a Detective Police-Officer, by Waters, London, Dabton \& Hodge, 1852.

9 "Detective Police" (Household Words, 27 juillet et 10 août 1850), "Three Detective Anecdotes" (14 septembre 1850), "On Duty with Inspector Field" (14 juin 1851), "Down with the Tide" (5 février 1853). Repris dans Reprinted Pieces, Londres, Chapman and Hall, 1859.

${ }^{10}$ DE BALZAC: Honoré Le Père Goriot, Paris, Gallimard, 1979, p. 279.

${ }^{11}$ FEVAL, Paul: Les Habits noirs, t. 1 (1865), réédition Paris, Laffont, 1987, p. 160.

${ }^{12}$ VAREILLE, Jean-Claude: L'Homme masqué, le détective et le justicier, Lyon, PUL, 1989, p. 108.
} 


\subsection{Formalisations}

Tout est donc en place vers le milieu du siècle pour que développe ce que Pierre Larousse appelle un peu plus tard «les romans de la cour d'assises et des investigation policière» ${ }^{13}$. L'invention romanesque du policier et l'imaginaire de la traque urbaine y ont renouvelé la fascination traditionnelle pour le crime. Une combinatoire narrative spécifique en résulte, dont la mise en forme progressive est aux sources du roman policier. Deux traits la caractérisent: la prise en main du récit par un «investigateur», la substitution du récit du crime par celui de l'enquête, dont le cheminement consiste précisément à reconstituer, par bribes et tâtonnements successifs, le méfait initial ${ }^{14}$. Un tel modèle, formalisé à l'aune très épurée du detective novel britannique des années 1920, n'existe cependant nulle part en l'état au XIXe siècle. Si les célèbres nouvelles publiées par Edgar Poe en 1842 purent constituer un prototype, ce que les contemporains perçurent comme des «romans d'investigations policières» étaient des récits composites et hybrides, mixte de fait divers, de mélodrame et de ténébreuses intrigues, mais où l'enquête judiciaire et la recherche de la vérité assuraient vaille que vaille la cohérence de l'ensemble.

Les mémoires de policiers, qui se multiplient en France comme en Angleterre dans la seconde moitié du siècle, participent clairement de ce mouvement. Récusant les «ratiocinations» fantaisistes, leurs mises en scène privilégient l'exercice de la police judiciaire et son travail de terrain ${ }^{15}$. Loin de toute abstraction intellectuelle elles aussi, les grandes séries criminelles façon Féval ou Ponson du Terrail perpétuent la veine feuilletonesque, mais la nourrissent d'éléments judiciaires et participent ainsi à l'avènement de ce roman de l'enquête. Y contribuent surtout les sensationnal novels que publie Wilkie Collins en Angleterre (The Women in White, 1860, The Moonstone, 1868 ) et les romans judiciaires que donne Émile Gaboriau (L'Affaire Lerouge, 1866, Le Crime d'Orcival, 1867). Dans les deux cas, le récit est clairement pris en charge par un agent de police judiciaire (le sergent Cuff chez Collins, les policiers Tabaret, puis Lecoq chez Gaboriau), et ordonné par la mise en œuvre d'une enquête chargée d'élucider le crime. Digressions, rebondissements, révélations, demeurent encore très présents, témoignant de l'emprise des traditions, mais une étape sensible est cependant franchie. En dépit de sa diversité, la production britannique s'oriente alors plus rapidement vers une formalisation du genre, que l'immense succès des romans et nouvelles de Conan Doyle accélère à compter de 1887, date de la première apparition publique de Sherlock Holmes ( $A$ Study in Scarlet). Même si prospèrent alors beaucoup de formes moins «achevées» (chez Arthur Morrison,

\footnotetext{
${ }^{13}$ Grand Dictionnaire universel du XIXe siècle, vol. XIII, p. 1327.

${ }^{14}$ Outre les ouvrages cités de Jacques Dubois et Jean-Claude Vareille, on reviendra à l'analyse classique de TODOROV, Tzvetan, «Typologie du roman policier», dans Poétique de la prose, Paris, Seuil, 1971, p. 10-19. Pour une bonne approche de type encyclopédique, voir KNIGTHS, Stephen: Crime Fiction 1800-2000: Detection, Death, Diversity, New York, Palgrave Macmillan, 2004.

${ }^{15}$ KALIFA, Dominique «Les mémoires de policiers: l'émergence d'un genre?», dans Crime et culture au XIXe siècle, Paris, Perrin, 2005, pp. 67-102.
} 
William Hornung ou Israël Zangwill), c'est dans l'Angleterre des années 1890 que se codifie le genre detective novel ${ }^{16}$.

La production française demeure plus hybride. Marquées par le vif essor du roman judiciaire (Adolphe Belot, Constant Guéroult, Pierre Zaccone, Fortuné du Boisgobey surtout), les années 1870 et 1880 rôdent des formules très composites où l'investigation policière travaille, sans la transformer complètement, la trame feuilletonesque. Celle-ci se prolonge sans grand effort jusqu'à la veille de la guerre, compliquée par les rejeux incessants du roman picaresque ou de la Bibliothèque Bleue (Arsène Lupin, Fantômas, Chéri-Bibi), la tentation du roman de détection (le cycle des Rouletabille de Gaston Leroux) ou l'influence, plus musclée, des dime novels venus d'outre-atlantique ${ }^{17}$. En résulte un ensemble disparate, en constante recomposition, mais dont beaucoup de contemporains voulurent voir l'unité, entre «roman criminel» et «roman d'aventures policières» $\rangle^{18}$. Le cœur en résidait dans l'exercice de l'enquête, principe actif chargé de remettre de l'ordre dans la société comme dans le texte.

\section{L'institution d'un imaginaire}

Portée par une littérature qui s'impose alors comme le principal pourvoyeur d'identités et de modèles sociaux et atteint, par l'intermédiaire de la presse et de la «libraire industrielle», des millions de lecteurs, cette prégnance du récit de l'enquête judiciaire nourrit un imaginaire dont les effets sont à la fois culturels, sociaux et politiques.

\subsection{Esprit scientifique?}

Dans un essai publié en 1929 et demeuré célèbre, Régis Messac insistait sur les liens unissant l'émergence du roman policier et le développement des sciences exactes ${ }^{19}$. Tant dans son principe que dans ses effets, le genre procédait selon lui des progrès du rationalisme et de la culture scientifique. Il soulignait notamment l'emprise de la physiognomonie, l'importance de la figure de Cuvier et du paradigme naturaliste, la vigueur du raisonnement inductif. Si d'autres sources étaient convoquées (le mythe d'Edipe, l'imaginaire picaresque ou celui de la roguery), l'éclairage décisif était porté sur l'essor du rationalisme, l'esprit du positivisme et l'influence d'une culture scientifique peu à peu diffusée dans les milieux urbains d'Europe occidentale. L'invention du roman de l'enquête traduirait et accompagnerait ainsi le mouvement général de vulga-

\footnotetext{
${ }^{16}$ EISENZWEIG, Uri Eisenzweig: Le Récit impossible. Forme et sens du récit policier, Paris, Bourgoin, 1989.

${ }^{17}$ DENNING, Michael, Mechanic Accent.Dime Novels and Working-Class Culture in America, London-New York, Verso, 1987.

${ }^{18}$ L'expression, plus tardive, est de Marcel ALLAIN, « Du roman populaire et de ses possibilités commerciales », 1938, repris Fantômas, Paris, Laffont, 1988, vol. 2, p. 1249.

${ }_{19}$ MESSAC, Régis Messac: Le Detective Novel et l'influence de la pensée scientifique, Paris, Champion, 1929.
} 
risation des sciences et d'éveil public au rationalisme. D'autres auteurs ont souligné les relations entre technologie, police scientifique et roman de détection ${ }^{20}$, insistant sur la nature expérimentale de récits qui ont toujours fait la part belle à la modernité technique (du Cour révélateur d'Edgar Poe en 1843, qui préfigure le sphygmographe et le détecteur de mensonge, aux usages précoces du télégraphe, du morse, du stéthoscope, de la dactyloscopie ou des pratiques d'identification et d'archivage).

Un tel mouvement d'ensemble est bien sûr indéniable. «La base du roman [est] déplacée et transportée du cœur à la tête et de la passion à l'idée», notent les Goncourt dans leur Journal du 16 juillet 1856, et il est clair que le récit d'enquête s'adosse à un régime de savoir (observation, induction, reconstitution) empreint de rationalisme et de positivisme. La multiplication des plans, des cartes et des indices textuels font du déchiffrement et de la raison graphique l'un des ressorts du genre. A tout le moins dans leur principe, expertise et herméneutique envahissent les codes de lecture et prônent la cérébralité comme valeur suprême. Dans Jean Diable, qu'il publie en 1862, Paul Féval met en scène le policier Gregory Temple, célèbre pour ses «calculs déductionnistes», qui couvre de chiffres, d'hypothèses et de probabilités un vaste tableau à multiples entrées ${ }^{21}$, et l'on sait combien le Sherlock Holmes de Conan Doyle, d'emblée promu en véritable icône du genre, incarne un tel esprit.

Le risque existe cependant de surestimer ces quelques exemples et de ne considérer la littérature criminelle du XIXe siècle qu'à l'aune d'un modèle - le roman de détection - tardif et marginal. L'immense majorité des récits d'enquête lus par les contemporains ne laisse au raisonnement qu'une place infime ou inexistante. Loin d'apparaître comme une mathématique capable de résoudre l'énigme dans un patient décryptage des indices et des traces, l'enquête y verse en permanence dans l'aventure, le coup de théâtre, la péripétie. L'emprise feuilletonesque y demeure dominante, condamnant souvent le raisonnement au profit l'événement, l'induction au profit du hasard. C'est notamment le cas en France, où le rationalisme policier peine à réformer une tradition «populaire» marquée par l'esprit carnavalesque, le goût de la transgression et le mélange des genres. La figure du ratiocinateur y est d'ailleurs fréquemment raillée, tant par les romanciers qui lui opposent le justicier ou l'aventurier (voire le criminel), que par les policiers qui insistent à l'inverse dans leurs mémoires sur l'empirisme et l'ordinaire ingrat du «travail de police».

Reste que les logiques de l'enquête, qui assurent la cohérence de l'ensemble, sont nécessairement porteuses d'une approche plus «éclairée». Les liens sont forts avec l'observation sociale, son souci d'exploration méthodique et de taxinomie. D'autres savoirs du temps y sont fréquemment convoqués: phrénologie, toxicologie, physiognomonie, anthropologie, faisant de la littérature un des relais majeurs des sciences criminelles, évoqué dès la fin du siècle par des criminalistes comme Enrico Ferri ou Scipio Shigele ${ }^{22}$. Une relation privilégiée se noua surtout presque d'emblée entre le roman d'enquête

${ }^{20}$ THOMAS, Ronald R: Detective Fiction and the Rise of Forensic Science, Cambridge University Press, 1999.

${ }^{21}$ FEVAL, Paul: Jean Diable, Paris, Dentu, 1863.

${ }^{22}$ FERRI, Enrico: Les Criminels dans l'art et la littérature, Paris, Alcan, 1895; Scipio Sighele, Littérature et criminalité, Paris, Giard et Brière, 1908. 
et l'image photographique, invention majeure du XIXe siècle. Adossés l'un comme l'autre au pouvoir d'identification et d'attestation du regard (la métaphore éculée de l'œil du policier!), ils partagent un même système de représentation qui entend bien «révéler» le monde à partir de l'enregistrement de ses traces sensibles. C'est pourquoi la fiction de l'œil révélateur ou de l'image inscrite dans la rétine comme dans L'Accusateur de Jules Claretie (1897) connurent une telle postéritéz ${ }^{23}$.

\subsection{Sociologie et hiérarchies romanesques}

En dépit de la diversité de ses formes, la littérature d'enquête œuvra presque mécaniquement à la promotion de nouvelles figures et au renouvellement du personnel romanesque. Le mouvement d'ensemble est évident, familiarisant les lecteurs avec les acteurs du processus judiciaire, policiers et magistrats en tête, concourrant de ce fait à l'acceptation progressive de professions jusque là perçues, et rejetées, comme de simples instruments d'un État prédateur. Même s'il ne fut ni général, ni continu, et transita aussi par bien d'autres canaux, ce procès de légitimation contribua à normaliser les représentations et les comportements.

$\mathrm{Au}$ cœur du processus réside la lente et progressive requalification du policier. Il est peu de dire que celui-ci constituait, au début du XIXe siècle, une figure honnie, oscillant de la silhouette sombre de l'espion à celle, moins retorse, mais tout aussi négative, du fonctionnaire incapable et obtus, englué dans la routine et la suffisance. Aux sources du phénomène se trouve, en France, la conception d'une police repensée et pratiquée par Fouché comme un instrument de renseignement et de basse politique. Maintenu par tous les régimes postérieurs, la figure du mouchard nourrit tout l'imaginaire romantique. Du Jackal de Dumas au Javert de Hugo, l'homme de police y demeure une figure froide et implacable, sur laquelle l'ombre portée de Vidocq surimprime encore la suspicion criminelle. «La honte et l'infamie l'enserrent de toutes parts, la société le chasse de son sein, l'isole comme un paria, lui crache son mépris avec sa paie, sans remords, sans regrets, sans pitié», lit-on en 1839 dans Les Français peints par eux-mêmes ${ }^{24}$. Et le rejet est tout aussi puissant en Grande-Bretagne, où la crainte de l'espionnage retarde jusqu'au milieu du siècle l'avènement d'une véritable police britannique.

En insistant sur la part honorable (c'est-à-dire judiciaire) de l'activité policière, en valorisant son rôle dans la construction de la «sécurité publique» et la défense des honnêtes gens, la littérature contribua donc activement à la requalification du métier. A cette utilité sociale s'ajouta la dimension culturelle et symbolique dont le personnage était investi. «Le policier, émanation de la société toute entière sous sa forme bureaucratique, devient alors la figure moderne de l'antique déchiffreur d'énigmes (Edipe),

${ }^{23}$ CAMPION-VINCENT, Véronique: «L'œil révélateur», Cahiers internationaux de sociologie, vol. CIV, 1998, pp. 55-75 ; GOULET, Andrea: Optiques. The Sciences of the Eye and the Birth of Modern French Fiction, Philadelphia, University of Pennsylvania Press, 2006.

${ }^{24}$ DURANTIN, Armand : «L'agent de la rue de Jérusalem», Les Français peints par eux-mêmes (vol. 2, 1839), rééd. Paris, Omnibus, 2003, p. 977. 
qui fait cesser le terrible pourquoi des choses; son activité, patiente et acharnée, est le symbole d'un désir profond : l'homme colmate fébrilement la brèche causale, il s'emploie à faire cesser une frustration et une angoisse», écrivait Roland Barthes ${ }^{25}$.

Il convient cependant de remarquer que ce mouvement, inégal selon les pays, est loin d'être achevé à la fin du XIXe siècle. Si de nombreux policiers se sont affirmés comme des figures romanesques de premier plan, il demeure difficile d'affirmer que «Sherlock Holmes a effacé la tache originelle hérité de Vidocq» ${ }^{26}$. A l'instar du Lestrade de Conan Doyle ou du Ganimard de Maurice Leblanc, le policier ordinaire manque d'éclat. S'il est sorti de la fange, il peine encore à s'imposer. C'est un fonctionnaire terne, rarement efficace, souvent ridicule. Grisaille! Ainsi se nomme par exemple l'inspecteur mis en scène par Fortuné du Boisgobey dans Décapitée, un roman judiciaire publié en 1889. La même grisaille recouvre souvent les autres acteurs de l'enquête officielle, notamment les juges d'instruction, qui ne parviennent pas à sortir de l'ornière. Si le ressort majeur est bien celui de l'investigation judiciaire, c'est à des enquêteurs privés qu'il revient le plus souvent de le mettre en action. Forte du modèle aristocratique, la tradition britannique valorise l'amateur éclairé, le détective dilettante. Même scénario en France, où les professionnels de l'investigation, toujours un peu suspects ou ridicules, doivent mettre chapeau bas devant quelque profane aux méthodes moins singulières, mais aux manières mieux assurées, justicier, aventurier ou simple quidam emporté par la passion de l'enquête. Le reporter s'impose surtout à la fin du siècle, en lien avec les progrès et surtout les ambitions du journalisme. Il sait «dénicher une affaire, écrit Fernand Xau en 1881, en suivre la piste, sans facilité, non pas avec la police, mais à côté d'elle, souvent même contre elle»» ${ }^{27}$. La Belle Époque en fera l'investigateur suprême, liant le reportage, l'enquête judiciaire et le roman de police sous les traits juvéniles du brillant Rouletabille ${ }^{28}$.

\subsection{Appropriations}

Cette importance attribuée à l'enquête officieuse renvoie aux pratiques de lecture qui furent celles de récits produits et consommés, à compter du milieu du XIXe siècle, sur une échelle industrielle. Cette question, on le sait, inquiéta les moralistes qui voulaient voir dans ces lectures un ferment majeur de corruption et de démoralisation $^{29}$. Les principaux effets sociaux et culturels semblent pourtant avoir été d'un autre ordre. Lus par un public de plus en plus nombreux, ces récits ont débord diffusé dans toutes les couches sociales une connaissance précise de la machinerie judiciaire,

${ }^{25}$ BARTHES, Roland, «Structure du fait divers» (1962) dans Essais critiques, Paris, Seuil, 1981, p. 198.

${ }^{26}$ MESSAC, Régis, op. cit., p. 644.

${ }^{27}$ Le Voltaire, 12 janvier 1881.

${ }^{28}$ KALIFA, Dominique: L'Encre et le sang. Récits de crimes et société à la Belle Époque, Paris, Fayard, 1995; id. «Criminal Investigators at the Fin-de-siècle», Yale French Studies, n 108, 2005, pp. $36-47$.

${ }^{29} I d$. «Roman policier et moralité publique», Tapis-franc, revue du roman populaire, $\mathrm{n}^{\circ} 4,1991$, pp. $128-147$. 
de ses acteurs et de ses rouages. «L'analyse psychologique du criminel y est négligée et sacrifiée à la description des péripéties judicaires», note Enrico Ferri ${ }^{30}$. Cette acculturation par la littérature ne se contentait pas d'entretenir une familiarité croissante avec les enquêteurs, les magistrats, les pièces à conviction et tout l'arsenal du savoir inquisitorial, elle en signalait aussi l'utilité sociale. Car si les voies étaient parfois sinueuses, l'issue, à quelques exceptions près, attestait toujours du triomphe de la loi et de l'ordre. Hier prédatrice, l'institution judiciaire se muait désormais en instrument de préservation sociale, en garant de la sécurité publique. Au travers de ces récits «policiers» se jouait ainsi un acte non négligeable du procès de normalisation sociale. Leur rôle était d'autant plus important qu'aucune enquête ne peut vraiment fonctionner hors du récit qui la porte; seule sa dimension narrative et son existence comme énoncé (procès-verbal, acte d'accusation ou réquisitoire de l'avocat général) permet à l'enquête judiciaire de prendre corps. D'où l'importance d'une littérature redoublant ces représentations pour les inscrire dans l'ordre de la fiction. Le phénomène traduisait, comme l'avait noté Michel Foucault ${ }^{31}$, «l'appropriation de la criminalité sous des formes recevables», dissociant les classes populaires d'un illégalisme projeté dans la seule dimension de la lecture et du spectacle.

Mais cette analyse en termes de pacification et de contrôle social n'épuise pas les significations du phénomène. Même s'il s'agit pour une large part d'un artifice, la littérature d'enquête repose sur l'idée d'une lecture active et participative. Tendus vers l'aval du récit, semés d'indices et ponctués de questions, ces récits invitaient le lecteur à collaborer à la résolution de l'énigme. La multiplication dans les journaux ou les fascicules de primes, de loteries, de concours, explicitement fondés sur la recherche d'indices textuels, en aiguisait encore l'intérêt. Pleinement constitué à la fin du XIXe siècle, tout un imaginaire fait de traces de pas, d'empreintes sanglantes et de messages mystérieux semblait convier la société à une intense activité de décryptage et d'interprétation. Sans doute cette frénésie relevait-elle pour partie de la caricature. Elle exprimait cependant, sur le mode de l'excès et de l'exubérance, cette obsession du déchiffrement qui taraude tout le XIXe siècle, et dont les facteurs sont sociaux autant que politiques.

Car la justice, on le sait, incarne une part importante de la légitimité du pouvoir. En collaborant aux côtés des enquêteurs à la résolution de l'énigme, le lecteur entend prendre sa part symbolique du pouvoir judiciaire. Le jury, mythe démocratique par excellence, semble s'étendre par ce biais à l'ensemble du corps social. L'imaginaire de l'enquête judiciaire travaille ainsi comme une fiction de la démocratie, contribuant à intégrer ses lecteurs dans la rationalité policée d'une société pacifiée. La diffusion sociale du genre accentue ce phénomène. De par ses origines et ses modalités d'expansion, le roman de l'enquête occupe un espace intermédiaire, à mi-chemin entre l'univers des littératures dominées et celui des avant-gardes, qui apprécient sa force d'innovation et de perturbation. Il est, selon l'expression de Jacques Dubois, un «agent double dans le champ littéraire» ${ }^{32}$. Son lectorat, de fait, transcende les catégories trop tranchées, liant

\footnotetext{
${ }^{30}$ FERRI, Enrico op. cit, p.71.

${ }^{31}$ FOUCAULT, Michel: Surveiller et punir. Naissance de la prison, Paris, Gallimard, 1975, pp. 68-72.

${ }^{32}$ DUBOIS, Jacques: «Un agent double dans le champ littéraire: le roman policier», Pratique, ${ }^{\circ}$ 50, 1986, p. 22-30.
} 
la middle class victorienne aux adolescents des faubourgs, les commis de province aux artistes de la capitale. "Un seul genre littéraire trouve aujourd'hui son public dans toutes les classes de la société, indifféremment: c'est le roman policier», écrivait en 1935 Jean-Richard Bloch dans L'Encyclopédie française $e^{33}$. C'était bien noter cette propension démocratique dont le roman de l'enquête est porteur.

${ }^{33}$ Cité par TREBITSCH, Michel: «Jean-Richard Bloch et la défense de la culture», Sociétés \& Représentations, $\mathrm{n}^{\circ} 15,2002$, p. 176. 